\title{
Robotic-assisted gait training applied with guidance force for balance and gait performance in persons with subacute hemiparetic stroke
}

\author{
Dong-Wook Son ${ }^{a}$, Sujin Hwang ${ }^{b}$ \\ ${ }^{a}$ Department of Physical Therapy, Graduate School of Health and Welfare, Baekseok University, Seoul, Republic of Korea \\ ${ }^{b}$ Department of Physical Therapy, Division of Health Science, Baekseok University, Cheonan, Republic of Korea
}

\begin{abstract}
Objective: Robot assisted gait training is implemented as part of therapy for the recovery of gait patterns in recent clinical fields, and the scope of implications are continuously increasing. However clear therapy protocols of robot assisted gait training are insufficent. The purpose of this study was to investigate the effects of robot-assisted gait training applied with guidance force on balance and gait performance in persons with hemiparetic stroke.
\end{abstract}

Design: Two group pre-test post-test design.

Methods: Nineteen persons were diagnosed with hemiparesis following stroke participated in this study. The participants were randomly assigned to the unilateral guidance group or bilateral guidance group to conduct robot-assisted gait training. All participants underwent robot-assisted gait training for twelve sessions ( $30 \mathrm{~min} / \mathrm{d}, 3 \mathrm{~d} /$ wk for 4 weeks). They were assessed with gait parameters (gait velocity, cadence, step length, stance phase, and swing phase) using Optogait. This study also measured the dynamic gait index (DGI), the Berg balance scale (BBS) score, and timed up and go (TUG).

Results: After training, BBS scores were was significantly increased in the bilateral training group than in the unilateral guidance group $(p<0.05)$. Spatiotemporal parameters were significantly changed in the bilateral training group (gait speed, swing phase ratio, and stance phase ratio) compared to the unilateral training group $(p<0.05)$.

Conclusions: The results of this study suggest that robot-assisted gait training show feasibility in facilitating improvements in balance and gait performance for subacute hemiparetic stroke patients.

Key Words: Gait, Postural balance, Stroke

\section{Introduction}

Stroke is one of the leading neurological diseases that result in acquired disabilities, and causes sudden and local neurological disabilities and unconsciousness for over 24 hours due to cerebrovascular accidents, such as cerebral infarction and cerebral hemorrhage [1]. Average life expectancy is increasing around the world along with the gradual increase of prevalence in stroke due to the greying societies. South Korea is not an exception to this global trend. Stroke is the third leading cause of death in adults just after cancers and cardiovascular diseases. Statistics Korea presented that there were 48 deaths per 100,000 people in 2015 [2].

Stroke survivors acquire not only motor and sensory dysfunctions, but also cognitive dysfunction, resulting in hemiplegia, abnormal muscle tone changes, abnormal posture and joint movement controls, motor control decrease, abnormal motor strategies, sensory deficits, and more [3]. As mentioned above, stroke survivors acquire different disabilities after the injury in the central nervous system, and one factor that greatly affects activities of daily living and functional activities is gait dysfunction [4]. Retaining gait

Received: 27, August, 2017 Revised: 17 September, 2017 Accepted: 18 September, 2017

Corresponding author: Sujin Hwang

Department of Physical Therapy, Division of Health Science, Baekseok University, 76 Munam-ro, Dongnam-gu, Cheonan 31065 , Republic of Korea Tel: 82-41-550-2309 Fax: 82-41-550-2829 E-mail: ptsue@bu.ac.kr

(c) This is an Open-Access article distributed under the terms of the Creative Commons Attribution Non-Commercial License (http://creativecommons.org/licens es/by-nc/4.0) which permits unrestricted non-commercial use, distribution, and reproduction in any medium, provided the original work is properly cited.

Copyright ( 2017 Korean Academy of Physical Therapy Rehabilitation Science 
abilities among stroke survivors is an essential factor for their independent living. In reality, $70 \%$ to $75 \%$ of stroke patients have some sort of balance and gait dysfunctions, and approximately $20 \%$ reported that they are dependent on everyday activities [5]. Therefore, gait training is one of the main focuses in stroke rehabilitation since the acute stage.

Gait patterns after stroke shows distinct characteristics such as foot drop, circumduction, and more. These gait patterns in stroke patients not only affect the dynamic postural balance, but also lead to muscle fatigue, spasticity, associated reaction, etc., resulting in ineffectiveness in functional gait [6].

In the general clinical field, widely used methods of treatment for restoration of stroke patients are the Bobath method and proprioceptive neuromuscular facilitation. These methods are used for the purpose of strengthening the core muscles through visual, auditory, tactile, and proprioceptive stimulations and recovery of normal motor patterns, postural control, and task performance [7]. Another method that is widely used for improving gait function in stroke patients is treadmill gait training, and this is based on the motor learning theory. The motor learning theory argues that treadmill gait training has a positive effect on functional recovery $[8,9]$. Repetitive gait training using treadmills based on this theory is effective in motor learning, and it is reported that there are improvements in asymmetrical gait patterns $[9,10]$. In addition, patients who have involuntary movements or have severe muscle weaknesses may be trained using a suspension-supported treadmill, and it is also useful in training patients in their acute stage in a safe manner [11]. However in order for the patients to train in a normal gait pattern on the treadmill, substantial physical efforts from the therapists are required, training intensiveness and time duration are limited, and in cases of involuntary movements or severe muscle weaknesses, establishing a normal gait pattern is impossible despite the assistance from the therapists [12].

Robot-assisted gait training is developed to decrease the physical efforts of therapists, promote normal gait patterns, and increase the intensiveness and training durations [13]. Robot-assisted gait training promotes sensory input related to motor in stroke patients to promote normal gait patterns, and has the advantage of performing high intensity training during the early stage for patients with gait dysfunctions due to stroke [14]. Research related to robot-assisted gait training is actively in place and has resulted in providing evidence that robot-assisted gait training has positive effects in improving balance abilities in stroke patients [15], and im- proves overall gait abilities [16]. Robot-assisted gait training is implemented as a part of therapy for the recovery of gait patterns in recent clinical fields, and the scope of implications are continuously increasing [17].

Research on robot-assisted gait training is continuously being conducted. However, clear therapy protocols of robot-assisted training and evidences of controllable variables are scarce and this is in contrast to active research on different patient groups and implication methods.

Therefore, this study aims to investigate the effects of different types of robot assistance on gait, balance, and symmetry in hemiplegic patients due to stroke.

\section{Methods}

\section{Participants}

This study was conducted with 20 patients who were admitted to local rehabilitation hospital with the diagnosis of hemiplegia due to stroke. Ten participants were randomly assigned to hemiplegia focused training group and 10 were assigned to bilateral training group using the table of random sampling numbers. The participants were provided with the study purpose and process, and the study was approved by the Institutional Review Board of Baekseok University (IRB No. BUIRB-201511-HR-028). The inclusion criteria were as follows: (1) persons diagnosed with hemiplegia due to stroke where the onset is more than 3 months and less than 6 months, (2) persons who are able to perform gait for 10 meters or more regardless of the usage of assistive devices, (3) persons without any neurological pathology except for stroke, (4) persons who do not have any trauma or osteoarthritic pathological issues, or a history of such in either lower extremities, and (5) persons who scored 24 or higher in Korean mini mental state examination and are able to follow the instructions of the researcher (Table 1).

\section{Procedures}

This study was the pretest and posttest with control group design. Twenty participants who conducted on the inclusion criteria were corrected from local rehabilitation center in Seoul. They were assigned to either unilateral or bilateral training group, and conducted robot-assisted gait training for 4 weeks ( $30 \mathrm{~min}$ a day, 3 times for week). To analyze the therapeutic effects after training, this study measured three clinical measures including timed up and go test (TUG), Berg balance scale (BBS), and dynamic gait index (DGI) and also measured spatiotemporal parameters including gait 
velocity, stride length, cadence, step length, gait symmetry, and single support period and double support period at one day before and after the interventions.

Table 1. Common characteristics of participants

$(\mathrm{N}=19)$

\begin{tabular}{lccr}
\hline Variable & $\begin{array}{c}\text { Unilateral } \\
\text { training } \\
\text { group }(\mathrm{n}=9)\end{array}$ & $\begin{array}{c}\text { Bilateral } \\
\text { training } \\
\text { group }(\mathrm{n}=10)\end{array}$ & $\mathrm{t}(p)$ \\
\hline Age $(\mathrm{yr})$ & $48.6(6.7)$ & $52.3(9.3)$ & $0.958(0.352)$ \\
Height $(\mathrm{cm})$ & $168.8(9.1)$ & $166.6(7.4)$ & $-0.579(0.148)$ \\
Weight $(\mathrm{kg})$ & $71.9(9.1)$ & $63.9(12.9)$ & $-1.519(0.571)$ \\
$\begin{array}{c}\text { Post-stroke } \\
\text { duration }(\mathrm{mo})\end{array}$ & $4.3(1.2)$ & $4.4(1.3)$ & $0.184(0.856)$ \\
$\begin{array}{c}\text { Sex } \\
\text { (female/male) }\end{array}$ & $3 / 6$ & $2 / 7$ & - \\
$\begin{array}{c}\text { Hemorrhage/ } \\
\text { infarction }\end{array}$ & $6 / 3$ & $5 / 4$ & - \\
\hline
\end{tabular}

Values are presented as mean (SD) or number only.
During robot-assisted gait training, initial weight bearing ratio was set to $30 \%$, and the speed of gait was gradually increased from the initial speed of the initial evaluation (Figure 1).

\section{Outcome measures}

\section{Berg balance scale}

BBS was developed to investigate the postural control and risk of fall for the geriatric population by K. Berg. The tool is composed of 14 tasks that require maintaining of balance or the increase in level of difficulty of tasks in order to assess static and dynamic balance performance and fall risks. Each items range from 0 (none) to 4 (maximum) points and the total score is 56 [18].

Less than 20 means there is a high risk of fall, 21 to 40 means moderate risk of fall, and higher than 41 mean low risk of fall. Inter-rater and intra-rater reliability are reported to be intra-class correlation coefficient $=0.97$ and 0.98 [19].

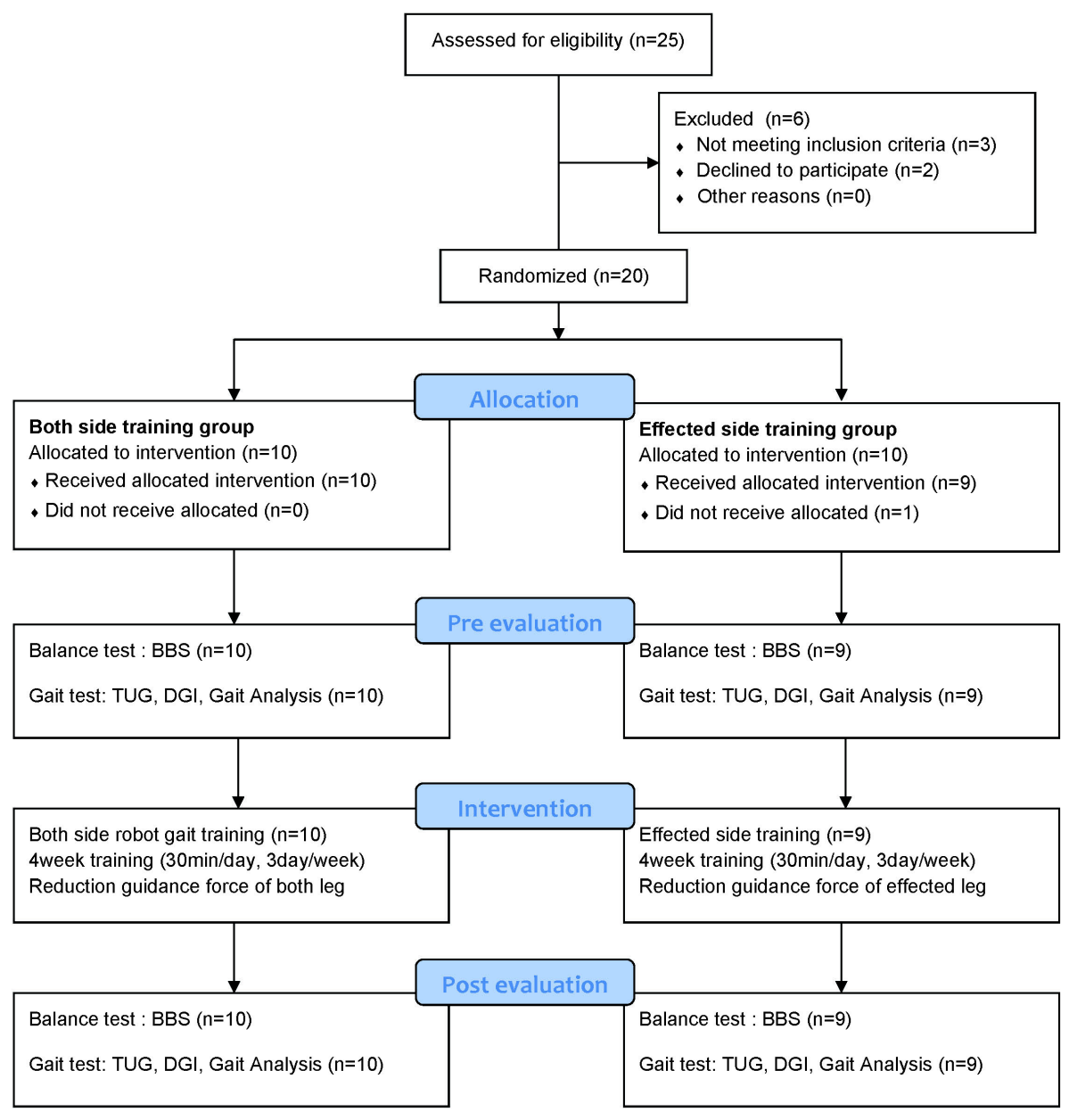

Figure 1. Flow diagram of the procedures in this study. BBS: Berg balance scale, TUG: timed up and go test, DGI: dynamic gait index. 


\section{Timed up and go test}

TUG was developed to assess anticipatory adjustments during gait performance. The participant sits on a chair with arm rests, and with the sign of the assessor, the time it takes for the participant to get up from the chair, walks 3 meters, and comes back to sit again is recorded. The participant may use assistive devices for gait, but should not receive and physical assistance. The normal range of the recorded time is less than 10 seconds, so the participants has abnormal dynamic balance and are dependent in physical mobility, if the time exceeds more than 20 seconds. This study measured three times and then used the mean value for analyzing. Inter-rater $(\mathrm{r}=0.99)$ and intra-rater reliability $(\mathrm{r}=0.98)$ for TUG are reported to be high [20].

\section{Dynamic gait index}

DGI was developed to evaluate individual's ability to modify balance while walking in the presence of external demands. The participant performs with a marked distance of 20 feet and can be performed with or without an assistance device. The tool is composed of 8 items such as steady state walking, walking with changing speeds, walking with head turns both horizontally and vertically, walking while stepping over and around obstacles, pivoting while walking, and stair climbing. The scores of the DGI are based on a 4-point scale from 0 (severe impairment) to 3 (no gait dysfunction), and highest possible score is 24 points. Lower than 19 indicates increased risk of falls in community-dwelling older adults and vestibular disorders. The tool has been showed a excellent test-retest reliability [21].

\section{Spatiotemporal parameters of gait performance}

OptoGait (OptoGait, Microgate, Bolzano, Italy) was used to analyze gait patterns of the participants. The gait analysis device is composed of sending and receiving a bar that is 1-meter length, with the installation of 5 meters with 2 meters of distance on a flat surface. Sending and receiving bar communicates with infrared light signals via installed light emitting diode. Lower extremities of the participant are sensed when the participant passes through the bar, and data regarding gait parameters are collected. Video information is saved in order to accurately synchronize starting foot order, errors due to overlapping of feet, and assessed gait. Items assessed for gait analysis are stance phase, swing phase, stance, velocity, cadence and assessed gait variables were processed through OptoGait1.5 (OptoGait).

\section{Statistical analysis}

The index for balance of the participants were derived from TUG and BBS scores, and gait ability used DGI scores, gait velocity, stride length, cadence, and step length from the gait analysis. Independent $t$-test was done in order to determine the differences in variables of participant's properties, age, height, weight, and diagnosis date. Independent t-tests were used to examine the effects of robot-assisted gait training, and matching sample t-test for each group were made to see the effects of robot-assisted training on balance and gait performance. Before and after intervention discrepancies were compared, and independent t-test was done in order to check for the differences. Statistically significant level was set to $\alpha=0.05$, and all statistical analysis of collected data were made using PASW Statistics ver. 18.0 (IBM Co., Armonk, NY, USA).

\section{Results}

The BBS score was significantly increase between pre-test and post-test in both groups, but the BBS score of bilateral training group was not significantly increase after training compared with that of unilateral training group $(p<0.05)$. However, the TUG and DGI scores were not significantly different between group after training, although the TUG and DGI scores were significantly improved between pre-test and post-test in both groups. The changeable value in TUG showed $8.7 \pm 5.8$ seconds for bilateral training group and $7.8 \pm 7.7$ seconds for unilateral training group. The changeable value in DGI showed $4.5 \pm 3.2$ for bilateral training group and 3.7 \pm 3.0 for unilateral training group (Table 2).

This study analyzed the spatiotemporal parameter during gait performance each paretic side respectively. In less-affected side, spatiotemporal parameters were not significantly different between pre-test and post-test in unilateral training group, while stance phase and swing phase only were significantly different between pre-test and post-test in bilateral training group. However, the swing phase was significantly improvement after training in bilateral training group compared with unilateral training group (Table 3). In more-affected side, stance phase, swing phase and gait velocity were significantly improvement between pre-test and post-test in bilateral group, but spatiotemporal parameters were not significantly different between pre-test and post-test in unilateral training group. However, the swing phase and stride length were significantly improvement in 
Table 2. Comparison between pretest and posttest of clinical measures in both groups

$(\mathrm{N}=19)$

\begin{tabular}{|c|c|c|c|c|c|c|c|}
\hline \multirow[b]{2}{*}{ Variable } & \multicolumn{3}{|c|}{ Unilateral training group $(\mathrm{n}=10)$} & \multicolumn{3}{|c|}{ Bilateral training group $(n=9)$} & \multirow{2}{*}{$\begin{array}{c}\text { Between group } \\
\mathrm{t}(p)\end{array}$} \\
\hline & Pre-test & Post-test & $\begin{array}{l}\text { Within group } \\
\text { (p-value) }\end{array}$ & Pre-test & Post-test & $\begin{array}{l}\text { Within group } \\
(p \text {-value })\end{array}$ & \\
\hline BBS (score) & $41.0(7.6)$ & $46.7(5.3)$ & $(0.004)$ & $42.1(9.2)$ & $46.0(7.2)$ & $(<0.001)$ & $-0.911(0.376)$ \\
\hline TUG (sec) & 30.7 (11.9) & $21.5(6.9)$ & $(0.001)$ & $31.5(18.0)$ & $24.3(11.1)$ & $(<0.001)$ & $0.591(0.563)$ \\
\hline DGI (score) & $14.1(5.1)$ & $18.3(3.8)$ & $(0.005)$ & $14.4(4.7)$ & $18.1(2.8)$ & $(0.022)$ & $-0.388(0.703)$ \\
\hline
\end{tabular}

Values are presented as mean (SD).

BBS: Berg balance scale, TUG: timed up and go test, DGI: dynamic gait index.

Table 3. Spatiotemporal parameters of gait performance in the less-affected side in both groups

$(\mathrm{N}=19)$

\begin{tabular}{|c|c|c|c|c|c|c|c|}
\hline \multirow[b]{2}{*}{ Variable } & \multicolumn{3}{|c|}{ Unilateral training group $(n=10)$} & \multicolumn{3}{|c|}{ Bilateral training group $(n=9)$} & \multirow{2}{*}{$\begin{array}{c}\text { Between group } \\
\mathrm{t}(p)\end{array}$} \\
\hline & Pre-test & Post-test & $\begin{array}{l}\text { Within group } \\
(p \text {-value })\end{array}$ & Pre-test & Post-test & $\begin{array}{l}\text { Within group } \\
(p \text {-value })\end{array}$ & \\
\hline Stance phase $(\%)$ & $70.4(7.0)$ & $69.8(8.0)$ & $(0.817)$ & $71.3(6.7)$ & $64.3(7.7)$ & $(0.010)$ & $1.896(0.076)$ \\
\hline Swing phase (\%) & $31.8(7.7)$ & $28.2(9.2)$ & $(0.407)$ & $29.2(5.9)$ & $36.0(5.7)$ & $(0.024)$ & $-2.178(0.045)$ \\
\hline Stride length (cm) & $75.3(14.0)$ & $75.5(11.2)$ & $(0.935)$ & $70.9(17.9)$ & $74.5(19.7)$ & $(0.762)$ & $-0.279(0.784)$ \\
\hline Gait velocity (m/s) & $0.5(0.2)$ & $0.5(0.1)$ & $(0.521)$ & $0.4(0.2)$ & $0.4(0.2)$ & $(0.984)$ & $0.216(0.832)$ \\
\hline Cadence (step/min) & $81.1(25.1)$ & $83.5(18.5)$ & $(0.695)$ & $75.7(23.9)$ & $70.7(22.3)$ & $(0.715)$ & $0.512(0.616)$ \\
\hline
\end{tabular}

Values are presented as mean (SD).

Table 4. Spatiotemporal parameters of gait performance in the more-affected side in both groups

$(\mathrm{N}=19)$

\begin{tabular}{|c|c|c|c|c|c|c|c|}
\hline \multirow[b]{2}{*}{ Variable } & \multicolumn{3}{|c|}{ Unilateral training group $(\mathrm{n}=10)$} & \multicolumn{3}{|c|}{ Bilateral training group $(n=9)$} & \multirow{2}{*}{$\begin{array}{c}\text { Between group } \\
\mathrm{t}(p)\end{array}$} \\
\hline & Pre-test & Post-test & $\begin{array}{l}\text { Within group } \\
\quad(p \text {-value })\end{array}$ & Pre-test & Post-test & $\begin{array}{l}\text { Within group } \\
\text { (p-value })\end{array}$ & \\
\hline Stance phase $(\%)$ & $72.2(5.2)$ & $69.3(9.5)$ & $(0.433)$ & $66.9(7.0)$ & $72.5(9.0)$ & $(0.033)$ & $-2.072(0.055)$ \\
\hline Swing phase (\%) & $28.6(4.7)$ & $31.9(10.4)$ & $(0.405)$ & $34.1(6.0)$ & $27.5(6.9)$ & $(0.002)$ & $2.453(0.026)$ \\
\hline Stride length $(\mathrm{cm})$ & $75.9(2.0)$ & $76.5(12.2)$ & $(0.811)$ & $73.6(16.6)$ & $77.6(18.5)$ & $(0.137)$ & $-0.962(0.013)$ \\
\hline Gait velocity (m/s) & $0.5(0.2)$ & $0.5(0.2)$ & $(0.891)$ & $0.5(0.3)$ & $0.6(0.3)$ & $(0.004)$ & $-2.781(0.716)$ \\
\hline Cadence (step/min) & $79.2(19.8)$ & $78.1(18.9)$ & $(0.815)$ & $71.7(23.3)$ & $73.5(22.8)$ & $(0.786)$ & $-0.370(0.195)$ \\
\hline
\end{tabular}

Values are presented as mean (SD).

bilateral training group after training compared with unilateral training group (Table 4).

\section{Discussion}

Early physical mobilization following stroke should be performed for getting positive prognosis and for integrating community and social roles [22]. In regards to rehabilitation of stroke patients, decrease in balance and gait act as a major inhibiting factor for activities of daily living, and recovery of balance and gait abilities is an important goal to accomplish functional independence [23]. Dynamic postural control such as weight shifting and weight bearing in the more-af- fected lower limb would impact the improvement of gait performance. Treadmill gait training induces coordination and motor control of the lower extremities in persons with hemiparetic stroke [24].

Robot-assisted gait device was designed in a way to take gait training during the early stages such as acute and subacute stages of diseases and to allow the intensive gait training in repetitive more normal patterns with decreased physical efforts by the physical therapist $[16,25,26]$.

Robot-assisted gait training increases a tolerance of weight bearing on the more-affected side and muscle activation to promote enhanced gait ability [12]. This study was to investigate the effects of types of manual guidance in ro- 
bot-assisted gait training on balance and gait performance for subacute hemiparetic stroke. The robot-assisted gait training was applying both weight tolerance and shifting of the more-affected side to train long term with normal patterns [27]. A previous study on the application of robot assisted gait training for 6 weeks with stroke patients reported that it was effective in increasing gait endurance and gait ability [14].

The participants of this study conducted robot-assisted three times per week, for four weeks. The postural stability shows improvement after robot-assisted gait training in both types of manual guidance, although the postural stability show more increase in the bilateral training group by facilitated bilateral movements. Both groups showed significantly positive changes relative to pre-training in both TUG and DGI, but there was no significant change between the two groups. In the gait analysis of the more-affected side, bilateral training group showed significant changed in weight tolerance, weight shifting, and speed, but there was no significant change in more-affected side focused training group.

This study demonstrated gait training based on motor learning theory using robot-assisted gait equipment. As a result of gait training for 4 weeks by both groups, significant results for both balance and gait ability were able to be derived, and the difference between the two methods were discovered. Robot-assisted gait training promotes intensive repetitive learning and normal patterns, making it an approach method for the improvement of balance and gait ability for patients with stroke. The form of guidance force application during robot assisted gait training affects balance and gait abilities. The types of guidance force application in robot-assisted gait training may be considered as an intervention to enhance balance and gait performance for subacute hemiparetic stroke. The advantages of implementing repetitive movements in normal patterns would allow the physical therapists to put less effort and may be implemented at the early rehabilitation stages. This is thought to have beneficial effects in balance and gait performance, and guidance force application differences could affect changes in gait patterns of hemiplegic patients.

This study was conducted with 19 participants, where the number is not big enough to generalize all hemiplegia in stroke. In addition, not doing follow up observations after 4 weeks of intervention is a limitation, which do not reflect the differences of long term intervene. There will be a need to consider these limitations in future studies, especially the need for long-term studies regarding various forms of robot assisted training and various patient groups.

\section{Conflict of Interest}

The authors declared no potential conflicts of interest with respect to the authorship and/or publication of this article.

\section{References}

1. WHO Scientific Group on Epidemiology Etiology, Prevention of Periodontal Diseases World Health Organization. Epidemiology, etiology, and prevention of periodontal diseases: report of a WHO scientific group. Geneva: World Health Organization; 1978.

2. Statistics Korea. Annual Report on the Causes of Death Statistics [Internet]. Deajeon: Statistics Korea, 2015 [cited 2016 Feb 9]. Available from: http://kostat.go.kr/portal/korea/kor_nw/2/6/ 2index.bord.

3. Poli P, Morone G, Rosati G, Masiero S. Robotic technologies and rehabilitation: new tools for stroke patients' therapy. Biomed Res Int 2013;2013:153872.

4. Aizen E. Falls in patients with stroke. Harefuah 2014;153:195-8, 237.

5. Monahan FD, Phipps WJ. Phipps' medical-surgical nursing: health and illness perspectives. St. Louis: Elsevier Mosby; 2007.

6. Tokuno CD, Eng JJ. Gait initiation is dependent on the function of the paretic trailing limb in individuals with stroke. Gait Posture 2006;24:424-8.

7. Chen JC, Shaw FZ. Progress in sensorimotor rehabilitative physical therapy programs for stroke patients. World J Clin Cases 2014;2:316-26.

8. Pohl M, Mehrholz J, Ritschel C, Rückriem S. Speed-dependent treadmill training in ambulatory hemiparetic stroke patients: a randomized controlled trial. Stroke 2002;33:553-8.

9. Park HJ, Oh DW, Kim SY, Choi JD. Effectiveness of community-based ambulation training for walking function of poststroke hemiparesis: a randomized controlled pilot trial. Clin Rehabil 2011;25:451-9.

10. Moore JL, Roth EJ, Killian C, Hornby TG. Locomotor training improves daily stepping activity and gait efficiency in individuals poststroke who have reached a "plateau" in recovery. Stroke 2010;41:129-35.

11. Moseley AM, Stark A, Cameron ID, Pollock A. Treadmill training and body weight support for walking after stroke. Cochrane Database Syst Rev 2005;(4):CD002840.

12. Husemann B, Müller F, Krewer C, Heller S, Koenig E. Effects of locomotion training with assistance of a robot-driven gait orthosis in hemiparetic patients after stroke: a randomized controlled pilot study. Stroke 2007;38:349-54.

13. Kosak MC, Reding MJ. Comparison of partial body weight-supported treadmill gait training versus aggressive bracing assisted walking post stroke. Neurorehabil Neural Repair 2000;14:13-9.

14. Mayr A, Kofler M, Quirbach E, Matzak H, Fröhlich K, Saltuari 
L. Prospective, blinded, randomized crossover study of gait rehabilitation in stroke patients using the Lokomat gait orthosis. Neurorehabil Neural Repair 2007;21:307-14.

15. Swinnen E, Beckwée D, Meeusen R, Baeyens JP, Kerckhofs E. Does robot-assisted gait rehabilitation improve balance in stroke patients? A systematic review. Top Stroke Rehabil 2014;21:87100.

16. Chang WH, Kim YH. Robot-assisted therapy in stroke rehabilitation. J Stroke 2013;15:174-81.

17. Hsieh YW, Lin KC, Wu CY, Lien HY, Chen JL, Chen CC, et al. Predicting clinically significant changes in motor and functional outcomes after robot-assisted stroke rehabilitation. Arch Phys Med Rehabil 2014;95:316-21.

18. Berg KO, Wood-Dauphinee SL, Williams JI, Maki B. Measuring balance in the elderly: validation of an instrument. Can J Public Health 1992;83 Suppl 2:S7-11.

19. Berg K, Wood-Dauphinee S, Williams JI. The balance scale: reliability assessment with elderly residents and patients with an acute stroke. Scand J Rehabil Med 1995;27:27-36.

20. Podsiadlo D, Richardson S. The timed "Up \& Go": a test of basic functional mobility for frail elderly persons. J Am Geriatr Soc 1991;39:142-8.
21. Shumway-Cook A, Baldwin M, Polissar NL, Gruber W. Predicting the probability for falls in community-dwelling older adults. Phys Ther 1997;77:812-9.

22. Arias M, Smith LN. Early mobilization of acute stroke patients. J Clin Nurs 2007;16:282-8.

23. Langhammer B, Stanghelle JK. Co-variation of tests commonly used in stroke rehabilitation. Physiother Res Int 2006;11:228-34.

24. Miller EW, Quinn ME, Seddon PG. Body weight support treadmill and overground ambulation training for two patients with chronic disability secondary to stroke. Phys Ther 2002;82:53-61.

25. Ochi M, Wada F, Saeki S, Hachisuka K. Gait training in subacute non-ambulatory stroke patients using a full weight-bearing gaitassistance robot: a prospective, randomized, open, blinded-endpoint trial. J Neurol Sci 2015;353:130-6.

26. Taveggia G, Ragusa I, Trani V, Cuva D, Angeretti C, Fontanella $\mathrm{M}$, et al. Robotic tilt table reduces the occurrence of orthostatic hypotension over time in vegetative states. Int J Rehabil Res 2015;38:162-6.

27. Brütsch K, Schuler T, Koenig A, Zimmerli L, -Koeneke SM, Lünenburger L, et al. Influence of virtual reality soccer game on walking performance in robotic assisted gait training for children. J Neuroeng Rehabil 2010;7:15. 